\title{
Infection after fracture fixation of the tibia: Analysis of healthcare utilization and related costs
}

\author{
Willem-Jan Metsemakers ${ }^{\mathrm{a}, \mathrm{c}, *}$, Bart Smeets $^{\mathrm{b}}$, Stefaan Nijs $^{\mathrm{a}, \mathrm{c}}$, Harm Hoekstra $^{\mathrm{a}, \mathrm{c}}$ \\ a University Hospitals Leuven, Department of Trauma Surgery, B-3000 Leuven, Belgium \\ ${ }^{\mathrm{b}}$ University Hospitals Leuven, Care Program Management, B-3000 Leuven, Belgium \\ ${ }^{\mathrm{c}} \mathrm{KU}$ Leuven, University of Leuven, Department of Development and Regeneration, B-3000 Leuven, Belgium
}

\section{A R T I C L E IN F O}

\section{Article history:}

Accepted 21 March 2017

\section{Keywords:}

Infection after fracture fixation

Complication

Cost analysis

Healthcare utilization

Deep infection

Tibia fractures

\begin{abstract}
A B S T R A C T
Introduction: One of the most challenging complications in musculoskeletal trauma surgery is the development of infection after fracture fixation (IAFF). It can delay healing, lead to permanent functional loss, or even amputation of the affected limb.

The main goal of this study was to investigate the total healthcare costs and length-of-stay (LOS) related to the surgical treatment of tibia fractures and furthermore identify the subset of clinical variables driving these costs within the Belgian healthcare system. The hypothesis was that deep infection would be the most important driver for total healthcare costs.

Patients and methods: Overall, 358 patients treated operatively for AO/OTA type 41, 42, and 43 tibia fractures between January 1, 2009 and January 1, 2014 were included in this study. A total of 26 clinical and process variables were defined. Calculated costs were limited to hospital care covered by the Belgian healthcare financing system. The five main cost categories studied were: honoraria, materials, hospitalization, day care admission, and pharmaceuticals.

Results: Multivariate analysis showed that deep infection was the most significant characteristic driving total healthcare costs and LOS related to the surgical treatment of tibia fractures. Furthermore, this complication resulted in the highest overall increase in total healthcare costs and LOS. Treatment costs were approximately 6.5-times higher compared to uninfected patients.

Conclusion: This study shows the enormous hospital-related healthcare costs associated with IAFF of the tibia. Treatment costs for patients with deep infection are higher than previously mentioned in the literature. Therefore, future research should focus more on prevention rather than treatment strategies, not only to reduce patient morbidity but also to reduce the socio-economic impact.
\end{abstract}

(C) 2017 Elsevier Ltd. All rights reserved.

\section{Introduction}

One of the most challenging complications in musculoskeletal trauma surgery is the development of infection after fracture fixation (IAFF). Infections associated with fracture fixation devices result in significant patient morbidity and a prolonged treatment period [1]. Currently, there is a lack of data regarding the definition, functional outcome, and healthcare burden of this sometimes devastating musculoskeletal complication.

Compared to prosthetic-joint infection (PJI) [2,3] or other fracture-related complications, such as nonunion [1,4], little data is available on the cost of implant-related infections in fracture care

\footnotetext{
* Corresponding author at: Department of Trauma Surgery, University Hospitals Leuven, Belgium.

E-mail address: willem-jan.metsemakers@uzleuven.be (W.-J. Metsemakers).
}

[5]. A better understanding of the healthcare burden of this serious complication is crucial. It will explain to healthcare institutions that future research is necessary and the development of preventive measures essential.

Although several studies have investigated healthcare costs of implant-related infections in orthopedic trauma surgery, most of these studies were heterogeneous, focused on an anatomical localization with a low incidence of infection (i.e., hip fractures), included a relatively small number of patients and most importantly, these studies were all performed in different countries [5-8]. Furthermore, one of the most cited publications by Darouiche is only descriptive without going into detail on patient inclusion and cost analysis regarding IAFF [9]. Currently, no study has provided a comprehensive analysis of the costs of treating IAFF among a large number of musculoskeletal trauma patients in Belgium or even other countries of the European mainland. Therefore, the main objective of this study was to define 
hospital-related healthcare costs associated with IAFF, more specifically deep infection of the tibia, and identify the subset of clinical variables relevant in driving these costs within the Belgian healthcare system. The choice for the tibia seemed logical as these fractures have a higher percentage of open injuries and risk for infection [10]. A recent publication indeed identified the tibia as an independent risk factor associated with IAFF [11].

\section{Patients and methods}

\section{Study and patient characteristics}

A total of 358 patients aged 18 years and over with either an acute AO/OTA type 41,42 , or 43 tibia fracture who underwent surgery between January 1, 2009 and January 1, 2014 were included in this study. All patients were primarily treated at the Traumatology Department of the University Hospitals Leuven, Belgium. Non-acute fractures (diagnosed more than 4 weeks after the incident), pathologic fractures, amputations of the affected limb within 5 days of the injury, age less than 18 years, and patients with multiple trauma were excluded. Clinical information was obtained from a retrospectively collected database (KWS; Klinisch WerkStation). Follow-up was until January 1, 2016, allowing a maximal follow-up time of two years for all patients. This study was conducted in compliance with national legislation and the guidelines of the ethics committee of the University Hospitals Leuven, Belgium. All tibia fracture-related hospital stays and ambulatory consults of the selected patients were included in the analysis. The authors adhered to the CHEERS Statement [12] and the CHEC-list [13] to ensure that all essential elements of the cost analysis were presented [14].

\section{Study aims}

- The first aim of this study was describe the total healthcare costs and length-of-stay (LOS) related to the surgical treatment of tibia fractures.

- The second aim was to evaluate which clinical and processrelated variables are relevant in driving total healthcare costs and LOS. We hypothesized that deep infection would be the most important clinical driver for total healthcare costs.

- The third aim was to describe the distribution of the 5 main cost categories for deep infection.

- The fourth aim was to perform a univariate comparison of process-related variables for patients without deep infection and with deep infection.

\section{Study variables}

A total of 26 characteristics were studied. Theses variables were grouped as clinical variables (gender, age, ASA-score, cardiovascular risk factors [CVRF], AO/OTA fracture classification, open fractures, delayed-stage surgery protocol, type of definite surgery, IAFF, nonunion, other tibia-related complications, debridement, hardware removal, re-osteosynthesis, and mortality) and processrelated variables (LOS to definite surgery, total length of antibiotic therapy, total LOS, number of operations, number of hospital admissions, number of surgical day care admissions, and number of ambulatory consults). CVRF included: age, current cardiovascular diseases (e.g., cerebrovascular event, myocardial infarction, peripheral artery disease), diabetes, rheumatoid arthritis, smoking, use of blood vessel narrowing drugs (e.g., beta blockers and ergotamine), dyslipidemia, hypercholesterolemia, and hypertension.
Based on X-rays or computed tomography (CT), if available, all fractures were classified to AO/OTA bone segment, fracture type, and fracture group [15]. Systemic prophylactic antibiotics were administered once before surgery for closed fractures and continued in case of open fractures until wound closure with a maximum of five days. Open fractures were subdivided by the Gustilo-Anderson classification [16], which was determined at the time of initial debridement in the operating room.

Delayed-stage surgery included all patients that were treated according to a delayed surgery protocol (i.e. external fixator prior to definite surgery). The type of definite surgery was categorized as intramedullary fixation, plate osteosynthesis, screw osteosynthesis, external fixator, or arthrodesis.

IAFF was classified into two groups, namely, superficial or deep infections, which were defined according to Dellinger et al. and Centers for Disease Control (CDC)-guidelines [17,18]. A superficial wound infection was one located above the fascia, with erythema and tenderness. A deep infection was defined as an infection involving deeper tissues as muscular fascia and bone, which could necessitate removal of the implant. Nonunion was assessed using follow-up radiographs and defined according to the US Food and Drug Administration (FDA) guidelines as a not completely healed fracture within 9 months of injury and without progression towards healing over the past 3 consecutive months [19]. Other tibia-related complications were wound problems, screw loosening, hardware migration, loss of reduction, peroneal nerve injury, and joint contracture. Debridement, hardware removal, reosteosynthesis, and other tibia-related operations were defined as secondary surgery; nearly all other tibia-related operations were bone grafting, (free) muscle flaps, and knee arthroplasty. LOS was defined as the number of consecutive hospital admission days during the stay for the definite treatment.

\section{Healthcare cost and cost categories}

The costs described in this paper relate to the Belgian healthcare financing system and are limited to costs related to hospital care. Furthermore, the costs investigated in this study are defined as the total reimbursements paid to the hospital by any party involved in financing the care for a specific patient either directly or indirectly. Five hospital-related cost categories were defined: honoraria, materials, hospitalization, day care admission, and pharmaceuticals. The honoraria category consists primarily of fees related to medical activities, based on a fee-for-service principle. These activities include: surgery, consults, and imaging. In the Belgian healthcare system, honoraria are independent of the rank of the surgeon as activities are billed under the attending physician. Material-related costs can be divided into costs related to the actual implants and those induced by extra-required materials (e.g. suture materials). Pharmaceuticals costs are all costs for received drugs and blood products. To analyze the performance impact due to differences in LOS, the patient's actual LOS was multiplied by the average national day based care fee (€410.84) [20]. The resulting sum was interpreted as the patient's hospitalization related costs.

\section{Statistical methods}

In order to determine the variables that drive total healthcare costs and LOS, three multivariate linear models were fitted to the data [21]. For all categorical variables, binary dummy variables were introduced to represent the categorical values. For each categorical variable, the number of dummy variables introduced equals the number of different values the categorical value can take minus one. Due to the large amount of potential independent variables a sequential forward search feature selection method 
was applied to each model in order to reduce the amount of independent variables with the objective to reduce statistical noise.

Categorical variables were compared with the Pearson ChiSquare test and presented as numbers and percentages. Comparison of continuous variables was performed using the MannWhitney $U$ test and presented as medians with interquartile range (IQR).

A 95\% confidence interval ellipse is used to visualize the interplay between total costs, age, and the presence of deep infection.

Exploratory data analysis and subsequent building of the statistical model was done using R and the MLR framework [22,23].

Table 1

Patient characteristics.

\begin{tabular}{|c|c|c|}
\hline & Patients & $\mathrm{n}=358$ \\
\hline \multirow{41}{*}{$\begin{array}{l}\text { Clinical } \\
\text { characteristics }\end{array}$} & \multicolumn{2}{|l|}{ Gender } \\
\hline & - male & 191 (53.4\%) \\
\hline & - female & $167(46.60 \%)$ \\
\hline & Age (years) & $49(36-62)$ \\
\hline & \multicolumn{2}{|l|}{ ASA score } \\
\hline & - ASA 1 & $158(44.1 \%)$ \\
\hline & - ASA 2 & $158(44.1 \%)$ \\
\hline & - ASA 3 & $42(11.7 \%)$ \\
\hline & CVRF & $198(58.9 \%)$ \\
\hline & \multicolumn{2}{|l|}{ AO/OTA bone segment } \\
\hline & -41 & $122(34.1 \%)$ \\
\hline & -42 & $140(39.1 \%)$ \\
\hline & -43 & $96(26.8 \%)$ \\
\hline & \multicolumn{2}{|l|}{ AO/OTA fracture type } \\
\hline & $-\mathrm{A}$ & $119(33.2 \%)$ \\
\hline & $-\mathrm{B}$ & $139(38.8 \%)$ \\
\hline & $-\mathrm{C}$ & $100(27.9 \%)$ \\
\hline & \multicolumn{2}{|l|}{ AO/OTA fracture group } \\
\hline & -1 & $162(45.3 \%)$ \\
\hline & -2 & $54(15.1 \%)$ \\
\hline & -3 & $142(39.7 \%)$ \\
\hline & Open fractures (Gustilo) & $51(14.2 \%)$ \\
\hline & $-\mathrm{I}$ & $26(51.0 \%)$ \\
\hline & - II & $15(29.4 \%)$ \\
\hline & - III & $10(19.6 \%)$ \\
\hline & Delayed-staged surgery & $44(12.3 \%)$ \\
\hline & \multicolumn{2}{|l|}{ Type of definite surgery } \\
\hline & - intramedullary nail & $140(39.1 \%)$ \\
\hline & - plate osteosynthesis & $144(40.2 \%)$ \\
\hline & - screw osteosynthesis & $66(18.4 .1 \%)$ \\
\hline & - external fixator & $7(2.0 \%)$ \\
\hline & - arthrodesis & $1(0.3 \%)$ \\
\hline & Superficial infection & $7(2.0 \%)$ \\
\hline & Deep infection & $12(3.4 \%)$ \\
\hline & Nonunion & $18(5.0 \%)$ \\
\hline & Other complications & $8(2.2 \%)$ \\
\hline & Debridement & $19(5.3 \%)$ \\
\hline & Hardware removal & $121(33.8 \%)$ \\
\hline & Re-osteosynthesis & $14(3.9 \%)$ \\
\hline & Other operations & $22(6.1 \%)$ \\
\hline & Mortality & $11(3.1 \%)$ \\
\hline \multirow{7}{*}{$\begin{array}{l}\text { Process } \\
\text { characteristics }\end{array}$} & LOS to definite surgery (days) & $1(0-3)$ \\
\hline & Total length of antibiotic therapy (days) & $1(1-3)$ \\
\hline & Total LOS (days) & $7(5-13)$ \\
\hline & Number of operations & $1(1-2)$ \\
\hline & Number of hospital admissions & $1(1-2)$ \\
\hline & Number of surgical day care admissions & 0 \\
\hline & Number of ambulatory consults & $5(3-8)$ \\
\hline
\end{tabular}

Clinical variables are presented as numbers and percentages, process variables as median with interquartile distributions. Abbreviations: CVRF, cardiovascular risk factors; LOS, length of stay.

\section{Results}

All clinical and process-related variables are described in Table 1 . Note that in total 12 patients (3.4\%) were diagnosed with a deep infection. In this group, 11 patients were male and one was female. Overall, there were 7 open (1 Gustilo type I, 3 Gustilo type II and 3 Gustilo type IIIB open fractures) and 5 closed fractures. The 3 patients with Gustilo type IIIB open fractures needed a free flap.

\section{Total healthcare costs and LOS for operative treatment of tibia fractures}

Overall, the total healthcare costs for the treatment of all 358 tibia fractures was calculated at $€ 3.713 .198$. This equates to a median total healthcare cost of $€ 6.962$ (IQR 4.932-10.972) per patient. The total LOS was calculated at 4628 days, which is equivalent to 7 days (IQR 5-13) per patient.

Deep infection is the most significant characteristic driving total healthcare costs and LOS related to operative treatment of tibia fractures

In order to determine the importance of both the clinical and process-related variables driving healthcare costs, three multivariate linear models were fitted to the data, using total healthcare costs as the dependent variable. The first model (Table 2, all variables costing model [AV-CM]) had an $R^{2}$ and adjusted $R^{2}$ of 0.97 . The variables nonunion, total LOS, number of hospital admissions, number of surgical day care admissions, and number of ambulatory consults were all deemed significant $(\mathrm{p} \leq 0.001)$.

However, the high predictive value of the first model is mostly due to the fact that the process-related variables (i.e. LOS) are inherently correlated to healthcare costs due to the financing system. Therefore, these non-stay related variables were excluded in a second model. As a consequence, the performance of this second model decreased significantly. The model had a $\mathrm{R}^{2}$ of 0.55 and an adjusted $\mathrm{R}^{2}$ of 0.53 (Table 2, non-stay-related costing model [NSRV-CM]). It shows the following significant variables ( $p$ $\leq 0.001$ ): deep infection, nonunion, age, ASA-3, and delayed-stage surgery.

Since total LOS was found the most important driver for healthcare costs $(t=81.81, A V-C M$, Table 2$)$, the question remains which clinical variables drive the LOS. Consequently, a third multivariate linear model was established using LOS as a dependent variable and the non-stay-related variables as independent variables. This model had a $\mathrm{R}^{2}$ of 0.48 and an adjusted $\mathrm{R}^{2}$ of 0.45 (Table 2, length-of-stay model [LS-M]). The variables deep infection, nonunion, age, ASA-3, and delayed-stage surgery were also found statistically significant $(\mathrm{p} \leq 0.001)$ in driving the LOS.

Distribution of healthcare costs for deep infection after operative treatment of tibia fractures

Operatively treated tibia fractures complicated by deep infection $(\mathrm{N}=12)$ resulted in the highest increase in total healthcare costs. The calculated median total healthcare cost for patients with a deep infection was 6.5 times higher compared to uninfected cases ( $€ 44.468$ [13.574-71.125] vs. €6.855 [4.899-10.495], respectively; Fig. 1). The distribution of the 5 main cost categories (honoraria, materials, hospitalization, daycare stay, and pharmaceuticals) and total costs for deep infection are summarized in Table 3. In total, $62 \%$ of the total healthcare cost, in cases complicated by a deep infection, was due to the hospitalization (e.g. LOS). Subsequently, the total LOS for deep infection was calculated at 777 days, which is equivalent to 54 days (IQR 19-111) per patient. 
Table 2

Multivariate linear models.

\begin{tabular}{|c|c|c|c|c|c|c|c|}
\hline & \multirow{3}{*}{$\begin{array}{l}\text { Model } \\
\text { Dependent variable } \\
\text { variable }\end{array}$} & \multirow{2}{*}{\multicolumn{2}{|c|}{$\frac{\mathrm{AV}-\mathrm{CM}}{\text { Total costs }}$}} & \multirow{2}{*}{\multicolumn{2}{|c|}{$\frac{\text { NSRV-CM }}{\text { Total costs }}$}} & \multirow{2}{*}{\multicolumn{2}{|c|}{$\frac{\text { LS-M }}{\text { Length-of-stay }}$}} \\
\hline & & & & & & & \\
\hline & & t-value & $\mathrm{p}$ & t-value & $\mathrm{p}$ & t-value & $\mathrm{p}$ \\
\hline \multirow[t]{50}{*}{ Clinical characteristics } & \multicolumn{7}{|l|}{ Gender } \\
\hline & - male & . & . & . & . & . & . \\
\hline & - female & . & . & -2.31 & $0.022^{*}$ & -2.37 & $0.018^{*}$ \\
\hline & Age & . & . & 3.81 & $<0.001^{* * *}$ & 4.43 & $<0.001^{* * *}$ \\
\hline & \multicolumn{7}{|l|}{ ASA score } \\
\hline & - ASA 1 & . & . & . & . & . & . \\
\hline & - ASA 2 & . & . & . & . & . & . \\
\hline & - ASA 3 & . & . & 4.10 & $<0.001^{* * *}$ & 3.69 & $<0.001^{* * *}$ \\
\hline & CVRF & . & . & 2.75 & $0.006^{* *}$ & 2.41 & $0.016^{*}$ \\
\hline & \multicolumn{7}{|l|}{ AO/OTA bone segment } \\
\hline & -41 & . & . & . & . & . & . \\
\hline & -42 & . & . & . & . & . & . \\
\hline & -43 & -1.43 & 0.152 & . & . & -1.12 & 0.263 \\
\hline & \multicolumn{7}{|l|}{ AO/OTA fracture type } \\
\hline & $-\mathrm{A}$ & . & . & . & . & . & . \\
\hline & $-\mathrm{B}$ & . & . & . & . & 1.54 & 0.125 \\
\hline & \multirow{2}{*}{\multicolumn{7}{|c|}{ AO/OTA fracture group }} \\
\hline & & & & & & & \\
\hline & -1 & . & . & . & . & . & . \\
\hline & -2 & . & . & . & . & . & . \\
\hline & -3 & . & . & . & . & . & . \\
\hline & \multicolumn{7}{|l|}{ Open fractures } \\
\hline & - Gustilo I & . & . & . & . & . & . \\
\hline & - Gustilo II & . & . & . & . & . & . \\
\hline & - Gustilo III & . & . & 2.30 & $0.022^{*}$ & 2.13 & $0.034^{*}$ \\
\hline & Delayed-staged surgery & . & . & 3.49 & $<0.001^{* * *}$ & 3.51 & $<0.001^{* * *}$ \\
\hline & \multicolumn{7}{|l|}{ Type of definite surgery } \\
\hline & - intramedullary nail & . & . & . & . & . & . \\
\hline & - plate osteosynthesis & . & . & -2.82 & $0.005^{* *}$ & -2.76 & $0.006^{* *}$ \\
\hline & - screw osteosynthesis & -3.26 & $0.0012^{* *}$ & -2.79 & $0.006^{* *}$ & -2.06 & $0.040^{*}$ \\
\hline & - external fixator & . & . & . & . & . & . \\
\hline & - arthrodesis & -0.91 & 0.364 & . & . & . & . \\
\hline & Superficial infection & -1.75 & 0.081 & . & . & . & . \\
\hline & Deep infection & . & . & 8.49 & $<0.001^{* * *}$ & 8.67 & $<0.001^{* * *}$ \\
\hline & Nonunion & 6.93 & $<0.001^{* * *}$ & 5.98 & $<0.001^{* * *}$ & 4.85 & $<0.001^{* * *}$ \\
\hline & Other complications & . & . & 1.67 & 0.095 & 1.70 & 0.090 \\
\hline & Debridement & . & . & . & . & . & . \\
\hline & Hardware removal & -1.84 & 0.067 & 1.72 & 0.086 & 1.46 & 0.147 \\
\hline & Re-osteosynthesis & 2.33 & $0.020^{*}$ & . & . & -1.96 & 0.050 \\
\hline & Other Operations & . & . & 1.72 & 0.087 & . & . \\
\hline & Mortality & . & . & . & . & . & . \\
\hline & LOS to definite surgery & . & . & excluded & excluded & excluded & excluded \\
\hline & Total length of antibiotic therapy & . & . & excluded & excluded & excluded & excluded \\
\hline & Total LOS & 81.81 & $<0.001^{* * *}$ & excluded & excluded & excluded & excluded \\
\hline & Number of operations & . & . & excluded & excluded & excluded & excluded \\
\hline & Number of hospital admissions & 5.97 & $<0.001^{* * *}$ & excluded & excluded & excluded & excluded \\
\hline & Number of surgical day care admissions & 4.30 & $<0.001^{* * *}$ & excluded & excluded & excluded & excluded \\
\hline & Number of ambulatory consults & 4.02 & $<0.001^{* * *}$ & excluded & excluded & excluded & excluded \\
\hline & $-\mathrm{R}^{2}$ & & 0.97 & & 0.55 & & 0.48 \\
\hline & $-\operatorname{Adj} R^{2}$ & & 0.97 & & 0.53 & & 0.45 \\
\hline
\end{tabular}

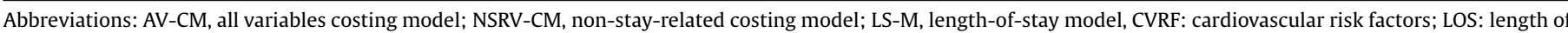
stay.

${ }^{*} \mathrm{p}<0.05^{* *} \mathrm{p}<0.01{ }^{* * *} \mathrm{p} \leq 0.001$ ( $\mathrm{p}<0.05$ is considered to be significant).

Total healthcare costs and LOS for nonunion, age, delayed-staged surgery and ASA-3

As compared to deep infection, patients treated for nonunion showed less increase in total healthcare costs (€6.773 [4.86810.470 ] vs. $€ 29.217$ [11.849-50.276], $\mathrm{p}<0.001$; union vs. nonunion) and LOS (7 [4-13] vs. 28 [12-67], p < 0.001; union vs. nonunion). The interplay between total healthcare costs, age, and presence of deep infection is shown in Fig. 2; although not statistically significant, there is a trend towards a positive correlation between age and total healthcare costs for deep infection. Patients treated according to a delayed-staged surgery protocol, revealed only a two-fold increase of the total healthcare costs (€6.545 [4.761-10.056] vs. €12.276 [8.025-19.167], p $<0.001)$ and LOS (6 [4-12] vs. 15 [9-28], p < 0.001; direct vs. delayed-stage surgery). Overall comorbidity (e.g. ASA) only lead to a limited increase in healthcare costs (€6.046 [4.597-9.097] vs. $€ 10.685$ [7.689-18.279)], $\mathrm{p}<0.001$; ASA-1 vs. ASA-3).

Description of process related-variables of deep infections - based patient cluster

A univariate comparison of process-related variables for patients without deep infection and with deep infection is shown in Table 4 . Note the statistically significant difference between 


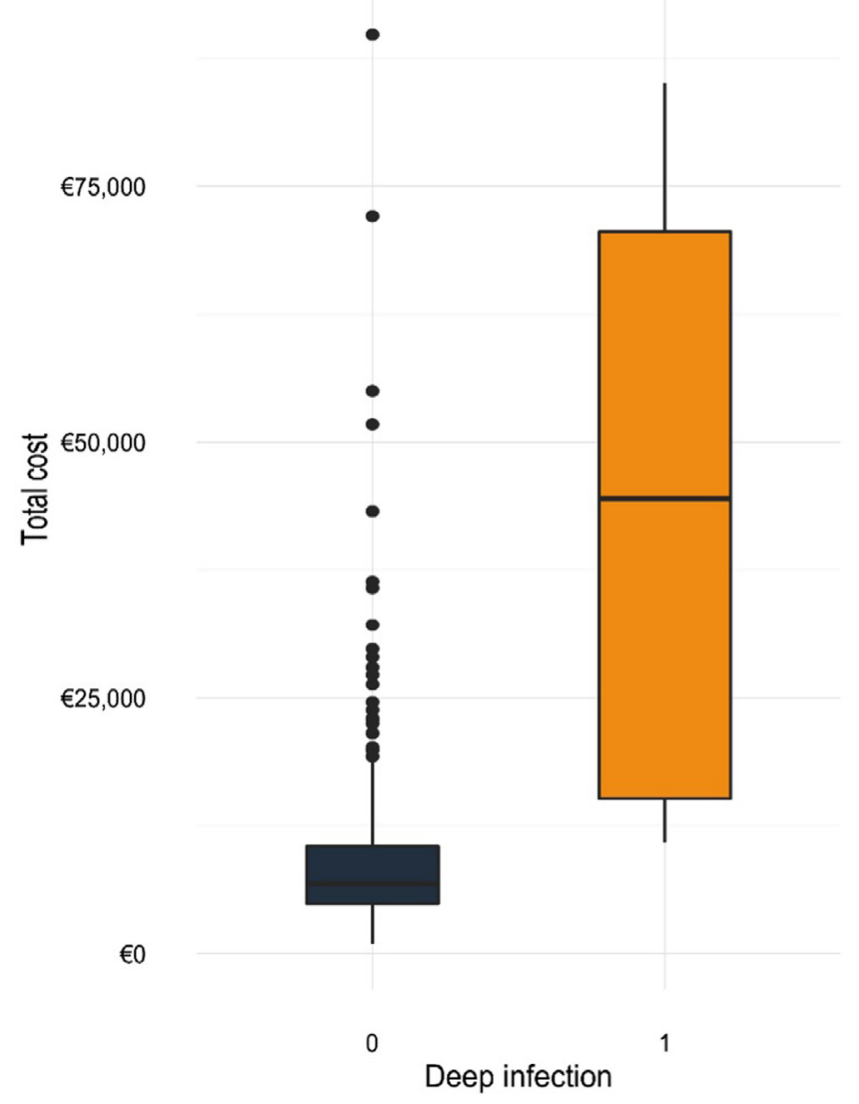

Fig. 1. Total healthcare costs for patients without and with deep infection.

infected and uninfected patients for almost all process-related variables.

\section{Discussion}

Musculoskeletal complications place a growing cost burden on total healthcare expenditure. One of the most challenging complications in trauma surgery is the development of IAFF. Despite research efforts, infection rates have been largely unchanged over the past decades [24]. Consequences for patients and healthcare systems regarding this complication are severe $[25,26]$.

The first aim of this study was to describe total healthcare costs and LOS related to the surgical treatment of tibia fractures. The second aim was to identify a subset of clinically relevant variables that drive these healthcare costs and LOS. We hypothesized that deep infection would be the most important driver for total healthcare costs. As previously mentioned, multivariate analysis showed that LOS was by far the most important driver for total healthcare costs $(t=81.81, A V-C M$, Table 2$)$. The question that

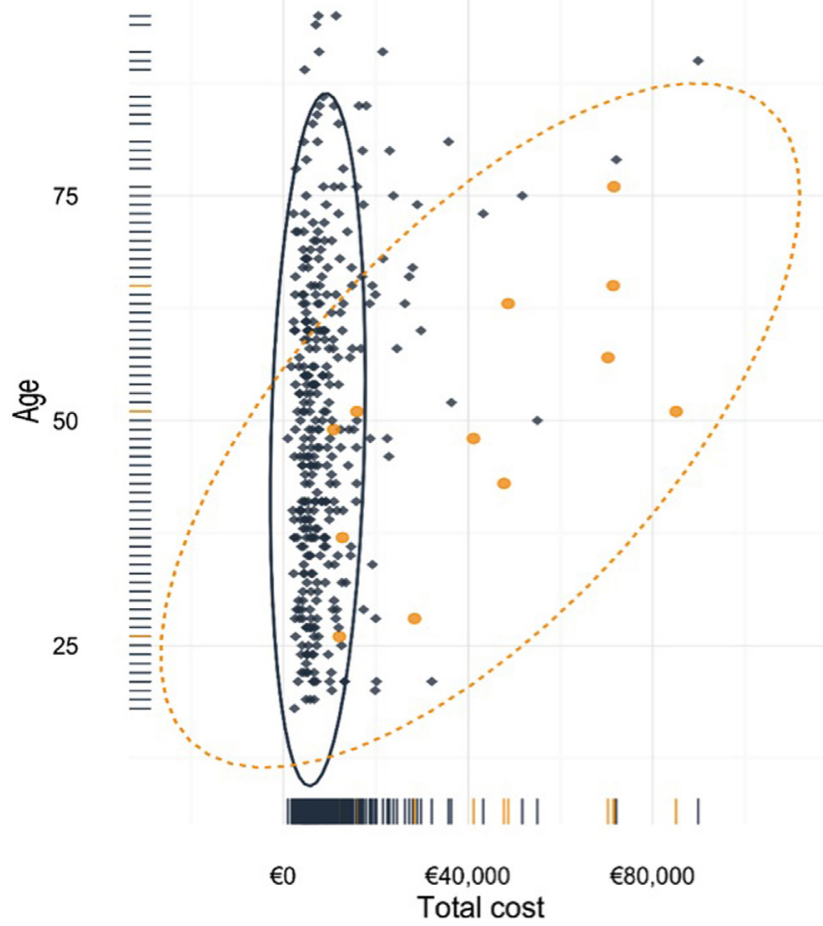

Deep infection $\rightarrow 0 \rightarrow 1$

Fig. 2. Interplay between total healthcare costs, age, and presence of deep infection.

remained was which clinical variables had the most significant impact on LOS. The length-of-stay model (LS-M; Table 2) revealed that the variables deep infection, nonunion, age, ASA-3, and delayed-stage surgery were found statistically significant ( $p$ $\leq 0.001$ ) in driving the LOS. Furthermore, when calculating the median total healthcare costs the results confirmed our hypothesis that tibia fractures complicated by deep infection resulted in the highest increase, compared to the other clinical variables (i.e. nonunion). More specific, treatment costs for infected patients were approximately 6.5-times higher compared to uninfected patients (€44.468 [13.574-71.125] vs. €6.855 [4.899-10.495], respectively; Fig. 1). This is much higher than previously published. As mentioned earlier, there are studies that describe the financial impact of IAFF in other countries [5,7,8,27]. Most of these studies show that infection doubles or triples healthcare costs. The most recent study by Olesen et al. compared healthcare costs for infected open tibia fractures treated with a free flap. The authors stated that infection increased the treatment costs from $€ 49.155$ in uninfected fractures to $€ 81.155$ for infected cases. Furthermore, infection increased the LOS from 41 to 74 days [28]. The reason why the overall treatment costs were higher in this study is probably due to two reasons. First the indirect costs (e.g. non-hospital-

Table 3

Healthcare costs for patients with deep infection $(n=12)$.

\begin{tabular}{|c|c|c|c|}
\hline Category & Per patient & Total & Relative share \\
\hline Honoraria & $€ 5.536(2.454-11.242)$ & $€ 78.315$ & $15 \%$ \\
\hline Materials (implants \& screws) & $€ 2.225(1.682-5.586)$ & $€ 42.720$ & $8 \%$ \\
\hline Hospitalization & $€ 22.185(7.909-45.501)$ & $€ 319.223$ & $62 \%$ \\
\hline Daycare stay & $€ 14(0-227)$ & $€ 2.087$ & $1 \%$ \\
\hline Pharmaceuticals & $€ 5.283(2.800-8.383)$ & $€ 73.698$ & $14 \%$ \\
\hline Total & $€ 44.468(13.574-71.125)$ & $€ 516.043$ & $100 \%$ \\
\hline
\end{tabular}

The costs per patient are presented as median followed by interquartile range. 
Table 4

Comparison process-related variables characteristics for deep infection.

\begin{tabular}{|c|c|c|c|}
\hline & $\begin{array}{l}\text { no deep infection } \\
(\mathrm{n}=346)\end{array}$ & $\begin{array}{l}\text { deep infection } \\
(\mathrm{n}=12)\end{array}$ & $\mathrm{p}$ \\
\hline LOS to definite surgery (days) & $1(0-3)$ & $4(0-10)$ & 0.076 \\
\hline Total length of antibiotic therapy (days) & $1(1-3)$ & $11(1-23)$ & $<0.001^{* * * *}$ \\
\hline Total LOS (days) & $7(4-13)$ & $54(19-111)$ & $<0.001^{* * *}$ \\
\hline Number of operations & $1(1-2)$ & $4(2-5)$ & $<0.001^{* * *}$ \\
\hline Number of hospital admissions & $1(1-2)$ & $3(2-5)$ & $<0.001^{* * * *}$ \\
\hline Number of surgical day care admissions & 0 & $0(0-1)$ & 0.455 \\
\hline Number of ambulatory consults & $5(3-7)$ & $10(6-15)$ & $<0.001^{* * *}$ \\
\hline
\end{tabular}

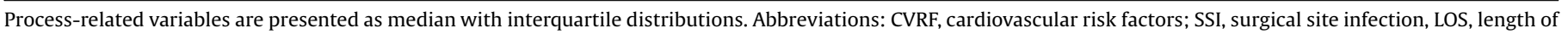
stay.

$\mathrm{p}<0.001$.

related costs) were included. Furthermore, the study only contained complex open tibia fractures treated with free flaps. These cases primarily need already more than one surgery. As mentioned earlier, our study contained only 3 patients with Gustilo type IIIB injuries that needed a free flap. Edwards et al. found that infection tripled overall costs and quadrupled LOS for hip fracture patients in the UK. In a study by Thakore et al., the authors reported that in orthopedic trauma, treatment costs were USD 108,782 in case of infection and USD 57,418 in uninfected patients [5]. This last publication showed some important differences compared to our study. First of all, the authors included multiple anatomical regions in a case-control study. Second, only the initial readmission following the primary admission after fracture fixation was included in the study. As shown in our study (Table 4), these readmissions are a major part of the overall healthcare cost. Therefore, the overall cost described by Thakore et al. is probably an underestimation. Furthermore, the study was performed outside Belgium, more specific in the USA. The importance of this issue becomes clear when looking at the total amount of costs for uninfected patients, these are higher compared to our results (USD 57,418 vs. USD $7671[€ 6.855]$ ). The fact that earlier research by Whitehouse et al. published lower treatment costs for uninfected patients in the USA (USD 6636 [€5.962]) [27], emphasizes the problem of heterogeneity in cost studies so far [14]. Due to the specificity of these national healthcare financing systems, it has to be noted that in order to make conclusions about generalizability of our study to other countries, other healthcare systems have to be comparable to the system in Belgium [21].

The third aim of our study was to describe the distribution of the 5 main cost categories for patients with a deep infection. As Table 3 shows, the higher costs related to deep infection were primarily attributable to long hospitalization periods (admissions and readmission), which accounted for approximately $62 \%$ of the overall healthcare costs. Indeed, when performing a univariate comparison of process-related variables for patients with deep infection and without deep infection (final aim of the study; Table 4), one of the most staggering results was the median number of hospitalization days. This difference was statistically significant when comparing both groups (54 (3 admissions) vs. 7 days ( 1 admission), respectively). As IAFF rates have remained unchanged over the past decades [24], we are aware that this complication will not be fully eradicated in the near future, which means that not only prevention but also reduction of treatment costs should be a topic of interest. To reduce these costs, focus should be on reduction of hospitalization days. Here implementation of standard protocols is mandatory. Primary issues in these protocols are often surgery related (e.g. fracture stability, wound care), but the antibiotic treatment can also prolong hospitalization, for this reason outpatient parenteral antibiotic therapy (OPAT) should always be considered in these protocols [29].
We realise that this study has limitations, as it is a retrospective analysis of suspected risk factors. Included data were limited to hospital costs related to tibia surgery and its complications. Other direct medical, direct non-medical, indirect, and psychosocial costs of IAFF were not included. Additionally, the deep infection subgroup ( $n=12$ ) was small; therefore, caution is needed before making statements regarding the results. Furthermore, the definition of IAFF is unclear [30]. The currently used CDCguidelines [18] (i.e., superficial and deep infection) were initially not developed for fracture patients. As mentioned by Bonnevialle et al., especially the term "superficial infection" is the least debatable [26]. We, therefore, stress that our definition of IAFF is arbitrary, and future specificity is warranted.

In conclusion we can state that for the first time, our study presents a comprehensive cost analysis, including "all" hospitalrelated costs, for treatment of deep infection of operatively treated tibia fractures (open and closed). The results show that the hospital-related healthcare cost of infected compared to uninfected cases is approximately 6.5-times higher, which is much higher than data that were previously published for other anatomical locations in other countries. This result stresses that future research should focus more on prevention rather than treatment strategies, not only to reduce patient morbidity but also to reduce the socio-economic impact. Finally, in the near future more multicenter analyses are needed to assess non-hospital-related, direct medical, direct non-medical, indirect, and psychosocial costs of IAFF. Including these costs will probably show higher overall healthcare costs than currently estimated, as shown by the study from Olesen et al. [28]. Furthermore, attention should be paid to the quality of cost analysis in orthopedic trauma and medicine in general, as current analyses are often heterogeneous and the available evidence variable [14,31].

\section{Conflict of interest}

All authors hereby disclose any financial and personal relationships with other people or organisations that could inappropriately influence this work.

\section{References}

[1] Trampuz A, Zimmerli W. Diagnosis and treatment of infections associated with fracture-fixation devices. Injury 2006;37(Suppl. 2):S59-66.

[2] Hellmann M, Mehta SD, Bishai DM, Mears SC, Zenilman JM. The estimated magnitude and direct hospital costs of prosthetic joint infections in the United States, 1997 to 2004. J Arthroplasty 2010;25:766-71 e1.

[3] Parvizi J, Pawasarat IM, Azzam KA, Joshi A, Hansen EN, Bozic KJ. Periprosthetic joint infection: the economic impact of methicillin-resistant infections. J. Arthroplasty 2010;25:103-7.

[4] Antonova E, Le TK, Burge R, Mershon J. Tibia shaft fractures: costly burden of nonunions. BMC Musculoskelet Disord 2013;14:42. 
[5] Thakore RV, Greenberg SE, Shi H, Foxx AM, Francois EL, Prablek MA, et al Surgical site infection in orthopedic trauma: a case-control study evaluating risk factors and cost. J Clin Orthop Trauma 2015;6:220-6.

[6] Kirkland KB, Briggs JP, Trivette SL, Wilkinson WE, Sexton DJ. The impact of surgical-site infections in the 1990: attributable mortality, excess length of hospitalization, and extra costs. Infect Control Hosp Epidemiol 1999;20:72530.

[7] Edwards C, Counsell A, Boulton C, Moran CG. Early infection after hip fracture surgery: risk factors, costs and outcome. J Bone Joint Surg Br 2008;90:770-7.

[8] Pollard TC, Newman JE, Barlow NJ, Price JD, Willett KM. Deep wound infection after proximal femoral fracture: consequences and costs. J Hosp Infect 2006;63:133-9.

[9] Darouiche RO. Treatment of infections associated with surgical implants. New Engl J Med 2004;350:1422-9.

[10] Papakostidis C, Kanakaris NK, Pretel J, Faour O, Morell DJ, Giannoudis PV. Prevalence of complications of open tibial shaft fractures stratified as per the Gustilo-Anderson classification. Injury 2011;42:1408-15.

[11] Bachoura A, Guitton TG, Smith RM, Vrahas MS, Zurakowski D, Ring D. Infirmity and injury complexity are risk factors for surgical-site infection after operative fracture care. Clin Orthop Relat Res 2011;469:2621-30.

[12] Husereau D, Drummond M, Petrou S, Carswell C, Moher D, Greenberg D, et al Consolidated Health Economic Evaluation Reporting Standards (CHEERS) statement. BMJ 2013;346:f1049.

[13] Evers S, Goossens M, de Vet H, van Tulder M, Ament A. Criteria list for assessment of methodological quality of economic evaluations: consensus on Health Economic Criteria. Int J Technol Assess Health Care 2005;21:240-5.

[14] Haddad FS, McLawhorn AS. Guidelines for reporting health economic research. Bone Joint J 2016;98-B:147-51.

[15] Marsh JL, Slongo TF, Agel J, Broderick JS, Creevey W, DeCoster TA, et al. Fracture and dislocation classification compendium - 2007: Orthopaedic Trauma Association classification, database and outcomes committee. J Orthop Trauma 2007:21:S1-133.

[16] Gustilo RB, Anderson JT. Prevention of infection in the treatment of one thousand and twenty-five open fractures of long bones: retrospective and prospective analyses. J Bone Joint Surg Am 1976;58:453-8.

[17] Dellinger EP, Miller SD, Wertz MJ, Grypma M, Droppert B, Anderson PA. Risk of infection after open fracture of the arm or leg. Arch Surg 1988;123:1320-7.

[18] Horan TC, Gaynes RP, Martone WJ, Jarvis WR, Emori TG. CDC definitions of nosocomial surgical site infections, 1992: a modification of CDC definitions of surgical wound infections. Infect Control Hosp Epidemiol 1992;13:606-8.
[19] Brinker MR. Nonunions: evalution and treatment. In: Browner BD, Levine AM, Jupiter JB, Trafton PG, editors. Skeletal Trauma: Basic Science, Management and Reconstruction. 3rd ed. Philadelphia: W.B. Saunders; 2003. p. 507-604.

[20] Rijksinstituut voor ziekte -en invaliditeitsverzekering (RIZIV). Actuele verpleegdagprijzen, 2016. http://www.riziv.fgov.be/nl/themas/kostterugbetaling/door-ziekenfonds/verzorging-ziekenhuizen/Paginas/ verpleegdagprijzen-ziekenhuizen.aspx2016.

[21] Smeets B, Nijs S, Nderlita M, Vandoren C, Hoekstra H. Health care usage and related costs in fibular plating for AO type 44-B ankle fractures in a Belgian university hospital: an exploratory analysis. J Foot Ankle Surg 2016;55:535-41.

[22] Team RC: A language and environment for statistical computing. Vienna, Austria,R Foundation for Statistical Computing, 2013.

[23] Bischl B, Lang M, Richter J, Bossek J, Judt L, Kuehn T, et al. mlr: Machine learning in $\mathrm{R}$. $\mathrm{R}$ package version..

[24] Willey M, Karam M. Impact of infection on fracture fixation. Orthop Clin N Am 2016;47:357-64.

[25] Zoubos AB, Galanakos SP, Soucacos PN. Orthopedics and biofilm?what do we know? A review. Med Sci Monit: Int Med J Exp Clin Res 201218: RA89-96.

[26] Bonnevialle P, Bonnomet F, Philippe R, Loubignac F, Rubens-Duval B, Talbi A, et al. Early surgical site infection in adult appendicular skeleton trauma surgery: a multicenter prospective series. Orthop Traumatol Surg Res 2012:98:684-9.

[27] Whitehouse JD, Friedman ND, Kirkland KB, Richardson WJ, Sexton DJ. The impact of surgical-site infections following orthopedic surgery at a community hospital and a university hospital: adverse quality of life, excess length of stay, and extra cost. Infect Control Hosp Epidemiol 2002;23:183-9.

[28] Olesen UK, Pedersen NJ, Eckardt H, Lykke-Meyer L, Bonde CT, Singh UM, et al. The cost of infection in severe open tibial fractures treated with a free flap. Int Orthop 2016.

[29] Chapman AL, Dixon S, Andrews D, Lillie PJ, Bazaz R, Patchett JD. Clinical efficacy and cost-effectiveness of outpatient parenteral antibiotic therapy (OPAT): a UK perspective. J Antimicrob Chemother 2009;64:1316-24.

[30] Metsemakers WJ, Moriarty TF, Morgenstern M, Kuehl R, Borens O, Kates S, et al. Letter to the editor: new definition for periprosthetic joint infection: from the workgroup of the musculoskeletal infection society. Clin Orthop Relat Res 2016:474:2726-7.

[31] Nwachukwu BU, Schairer WW, O'Dea E, McCormick F, Lane JM. The quality of cost-utility analyses in orthopedic trauma. Orthopedics 2015;38:e673-80. 